Archives of Agriculture and Environmental Science

\title{
Influence of plant nutrient management on the yield performance of transplant Aman rice (Oryza sativa L.)
}

\author{
Md. Imran Ali, Md. Abdur Rahman Sarkar and Swapan Kumar Paul* \\ Department of Agronomy, Bangladesh Agricultural University, Mymensigh-2202, BANGLADESH \\ "Corresponding author's E-mail: skpaul@bau.edu.bd
}

\section{ARTICLE HISTORY}

Received: 21 January 2018

Revised received: 27 January 2018

Accepted: 18 February 2018

\section{Keywords}

Nutrient management

Plant nutrient management

Transplant Aman rice

Variety

Yield

\section{ABSTRACT}

The experiment was conducted at the Agronomy Field Laboratory, Bangladesh Agricultural University, Mymensingh, Bangladesh during June to December 2016 to investigate the influence of plant nutrient management on the yield performance of transplant Aman rice varieties. The experiment comprised four varieties viz., BRRIdhan70, BRRI dhan71, BRRI dhan72 and BRRIdhan73 and six nutrient managements viz. poultry manure $5 \mathrm{t} \mathrm{ha}^{-1}$, recommended dose of prilled urea, P, K, S, Zn (160, 65, 90, 70, $10 \mathrm{~kg} \mathrm{ha}^{-1}$ of urea, TSP, MoP, Gypsum and Zinc sulphate, respectively), $75 \%$ of recommended dose of prilled urea and $\mathrm{P}, \mathrm{K}, \mathrm{S}, \mathrm{Zn}+$ poultry manure 2.5 t ha' ${ }^{1}, 50 \%$ of recommended dose of prilled urea and $P, K, S, Z n+$ poultry manure $5 \mathrm{t} \mathrm{ha}^{-1}$, USG $1.8 \mathrm{~g} / 4$ hills and $\mathrm{P}, \mathrm{K}, \mathrm{S}, \mathrm{Zn}$ recommended dose, USG $1.8 \mathrm{~g} / 4$ hills and $\mathrm{P}, \mathrm{K}, \mathrm{S}, \mathrm{Zn}+$ poultry manure $2.5 \mathrm{t}$ ha ${ }^{-1}$. The experiment was laid out in a randomized complete block design with three replications. Number of total tillers hill $^{-1}(10.25)$, number of effective tillers hill ${ }^{-1}(8.85)$, grains panicle ${ }^{-1}$ (94.23), 1000-grain weight (27.81), grain yield (5.88 $\mathrm{t} \mathrm{ha}^{-1}$ ) and straw yield (8.83 $\mathrm{t} \mathrm{ha} \mathrm{h}^{-1}$ ) were found to be the highest in BRRI dhan72. Among the nutrient management, USG $1.8 \mathrm{~g} / 4$ hills and $\mathrm{P}, \mathrm{K}, \mathrm{S}, \mathrm{Zn}+$ poultry manure $2.5 \mathrm{t} \mathrm{ha}^{-1}$ exhibited its superiority to other treatments in terms of plant height $(131.0 \mathrm{~cm})$, number of total tillers hill $^{-1}(10.67)$, number of effective tillers hill ${ }^{-1}$ (9.13), grains panicle ${ }^{-1}$ (92.71), 1000-grain weight (26.82), grain yield (6.0 $\mathrm{t} \mathrm{ha}^{-1}$ ) and straw yield $\left(8.35 \mathrm{t} \mathrm{ha}^{-1}\right)$. The highest grain yield $\left(6.45 \mathrm{tha}^{-1}\right)$ was found in BRRI dhan72 combined with USG $1.8 \mathrm{~g} / 4$ hills and $\mathrm{P}, \mathrm{K}, \mathrm{S}, \mathrm{Zn}+$ poultry manure $2.5 \mathrm{t} \mathrm{ha}^{-1}$ and the lowest grain yield $\left(4.85 \mathrm{t} \mathrm{ha}^{-1}\right)$ was found in BRRI dhan71 fertilized with poultry manure $5 \mathrm{t} \mathrm{ha}^{-1}$. From the study, it can be concluded that transplant Aman rice cv. BRRI dhan72 fertilized with USG $1.8 \mathrm{~g} / 4$ hills and P, K, S, Zn + poultry manure $2.5 \mathrm{tha}^{-1}$ appears as the promising practice to obtain the highest grain yield.

(C)2018 Agriculture and Environmental Science Academy

Citation of this article: Ali, M.I., Sarkar, M.A.R and Paul, S.K. (2018). Influence of plant nutrient management on the yield performance of transplant Aman rice (Oryza sativa L.). Archives of Agriculture and Environmental Science, 3(1): 49-53 DOI: 10.26832/24566632.2018.030106

\section{INTRODUCTION}

Rice (Oryza sativa L.) crop is interwoven in the cultural, social and economic lives of millions of Bangladeshis and it holds the key for food and nutritional security of the country. It is consumed as the staple food and has been given the highest priority in meeting the demands of ever-increasing population in Bangladesh. It is the most important food crop and a primary food source for more than one-third of world's population (Singh and Singh, 2008). In Bangladesh, it is grown under irrigated, rainfed and deep water conditions in the three distinct seasons namely;
Aus, Aman and Boro. Among the three distinct seasons Aman rice covers the second largest area of 56 lac hectares with a production of 131 lac tons of rice (BBS, 2016). In the recent years, crop productivity has stagnated or decreased in spite of consumption of increased rate of chemical fertilizers (Chen et al., 2011). As a result, agricultural ecosystems remain in a state of chemical nutrient saturation, leading to huge nutrient losses through leaching, runoff, volatilization, emissions, immobilization and subsequent low nutrient use efficiency (Sun et al., 2012). It is high time to search for innovative practices, which can guarantee higher yields with minimal deterioration of natural 
resources. Integrated nutrient management has been shown to considerably improve rice yields by minimizing nutrient losses to the environment and managing the nutrient supply, and thereby results in high nutrient use efficiency (Kumar and Yadav, 2008). Recent field experiments have demonstrated that integrated nutrient management can lead to significant increase in crop yields while substantially reducing nutrient losses (Gupta and Sharma, 2007). Strong and convincing evidence indicates that INM practice could be an innovative and environmentally friendly strategy for sustainable agriculture worldwide ( $\mathrm{Wu}$ and $\mathrm{Ma}$, 2015).Therefore, the present investigation was carried out to have a better understanding of improving nutrient management in transplant Aman rice for maximization of yield.

\section{MATERIALS AND METHODS}

The experiment was conducted at the Agronomy Field Laboratory, Bangladesh Agricultural University (BAU), Mymensingh, during June to December 2016 to study the influence of plant nutrient management on the yield performance of transplant Aman rice varieties. The land was medium high with silt-loam texture having $\mathrm{pH}$ 5.9. The experiment comprised four varieties viz. BRRIdhan70, BRRI dhan71, BRRI dhan72 and BRRIdhan73 and six nutrient managements viz. poultry manure $5 \mathrm{t} \mathrm{ha}^{-1}$, recommended dose of prilled urea, P, K, S, Zn (160, 65, 90, 70, $10 \mathrm{~kg}$ $\mathrm{ha}^{-1}$ of urea, TSP, MoP, Gypsum and Zinc sulphate, respectively), $75 \%$ of recommended dose of prilled urea and $\mathrm{P}, \mathrm{K}, \mathrm{S}, \mathrm{Zn}+$ poultry manure $2.5 \mathrm{t} \mathrm{ha}^{-1}, 50 \%$ of recommended dose of prilled urea and $\mathrm{P}, \mathrm{K}, \mathrm{S}, \mathrm{Zn}+$ poultry manure $5 \mathrm{t} \mathrm{ha}^{-1}$, USG $1.8 \mathrm{~g} / 4$ hills and $\mathrm{P}$, $\mathrm{K}, \mathrm{S}, \mathrm{Zn}$ recommended dose, USG $1.8 \mathrm{~g} / 4$ hills and $\mathrm{P}, \mathrm{K}, \mathrm{S}, \mathrm{Zn}+$ poultry manure $2.5 \mathrm{t} \mathrm{ha}^{-1}$. The experiment was laid out in a randomized complete block design with three replications. The size of unit plot was $4.0 \times 2.5 \mathrm{~m}$. Poultry manure, urea, TSP, MoP, gypsum and zinc sulphate were applied at final land preparation as per treatment requirements. Urea was top dressed in three equal splits at 15,30 and 45 days after transplanting (DAT). USG was applied at 8 DAT at the center of four hills in every alternate row. Prior to harvest, five hills plot ${ }^{-1}$ were randomly selected excluding border hills and central one square meter harvest area to record data on crop characters and yield components. The crop was harvested at full maturity and threshed by pedal thresher to record the fresh weight of grain and straw. Grains were cleaned and sun dried to a moisture content of $14 \%$. Straws were also sun dried properly. Grain and straw yields were then converted to $\mathrm{tha}^{-1}$. The recorded data were analyzed statistically using Analysis of variance and the mean differences among the treatments were adjudged by Duncan's Multiple Range Test (Gomez and Gomez, 1984).

\section{RESULTS AND DISCUSSION}

\section{Effect of variety}

Crop characters, yield components and yield of transplant Aman rice were significantly influenced by variety. BRRI dhan70 produced the tallest plant $(139.9 \mathrm{~cm})$ followed by BRRI dhan72
$(128.0 \mathrm{~cm})$ and BRRI dhan71 $(122.2 \mathrm{~cm})$ while the shortest one $(121.8 \mathrm{~cm})$ was recorded in BRRI dhan73. These differences were mostly due to the genetic variation among the varieties. These results are consistent to those of Pal et al. (2016) and Chowdhury et al. (2016) who reported variable plant height among the varieties. The highest number of total tillers hill ${ }^{-1}$ (10.25), number of effective tillers hill $^{-1}$ (8.85) and number of non-effective tillers hill $^{-1}$ (1.40) were recorded in BRRI dhan72 while the lowest values were found in BRRI dhan70. The longest panicle $(26.60 \mathrm{~cm})$ was found in BRRI dhan73 while the shortest one $(23.57 \mathrm{~cm})$ was recorded in BRRI dhan71 which was at par with BRRI dhan70 and BRRI dhan72. The highest number of grains panicle ${ }^{-1}$ (88.21) and 1000-grain weight (26.66 g) were found in BRRI dhan72 whereas the lowest values were recorded in BRRI dhan70. The highest grain yield $\left(5.88 \mathrm{t} \mathrm{ha}^{-1}\right)$ and straw yields (6.71 $\mathrm{t} \mathrm{ha}^{-1}$ ) were obtained in BRRI dhan72 followed by BRRI dhan73 while the lowest values were recorded in BRRI dhan70. The variation in number of tillers hill ${ }^{-1}$ as assessed might be due to varietal characters. Nuruzzaman et al. (2000) noticed that number of tillers hill ${ }^{-1}$ differed among the varieties. Varietal differences regarding grain yield was reported elsewhere (Tyeb et al. 2013; Jisan et al., 2014; Pal et al., 2016). The highest harvest index (45.00\%) was recorded in BRRI dhan70, which was at par with BRRI dhan71 and the lowest one in BRRI dhan72. Variety has significant influence on harvest index was also reported elsewhere (Tyeb et al., 2013; Sarkar et al., 2014 and Chowdhury et al., 2016).

\section{Effect of nutrient management}

Nutrient management significantly influenced crop characters, yield components and yield (Table 2). The application of USG 1.8 $\mathrm{g} / 4$ hills and $\mathrm{P}, \mathrm{K}, \mathrm{S}, \mathrm{Zn}+$ poultry manure $2.5 \mathrm{t} \mathrm{ha}^{-1}$ showed its superiority in terms of plant height $(131.0 \mathrm{~cm})$, number of total tillers hill ${ }^{-1}$ (10.67), number of effective tillers hill ${ }^{-1}$ (9.13) and grains panicle ${ }^{-1}$ (92.71) to nutrient management while the lowest values for these parameters were found in poultry manure 5 $\mathrm{t} \mathrm{ha}^{-1}$. The highest grain yield $\left(6.0 \mathrm{t} \mathrm{ha}^{-1}\right)$ and straw yield $(8.35 \mathrm{t}$ $\mathrm{ha}^{-1}$ ) were obtained when the crop was fertilized with USG 1.8 $\mathrm{g} / 4$ hills and $\mathrm{P}, \mathrm{K}, \mathrm{S}, \mathrm{Zn}+$ poultry manure $2.5 \mathrm{t} \mathrm{ha}^{-1}$. Probably this treatment might have provided adequate nutrients to plants and due to absorption of more nutrients, the crop produced the highest grain yield. These results are in agreement with that of Pal et al., 2016; Biswas et al., 2016; Islam et al., 2015; Roy et al., 2015 and Sarkar et al., 2014 who found differences in yield and yield components due to levels of nutrient management. The treatment poultry manure $5 \mathrm{t} \mathrm{ha}^{-1}$ gave the lowest values for the same parameters due to poor nutrient supply and its uptake by plant. Application of USG $1.8 \mathrm{~g} / 4$ hills and $\mathrm{P}, \mathrm{K}, \mathrm{S}, \mathrm{Zn}+$ poultry manure $2.5 \mathrm{t} \mathrm{ha}^{-1}$ improved the yield contributing characters viz. number of effective tillers hill ${ }^{-1}$, number of grains panicle ${ }^{-1}$ and 1000-grain weight, which ultimately resulted in the highest grain yield. The straw yield showed similar trend as that of grain yield due to nutrient management. Application of different doses of manures and fertilizers influenced the crop characters in terms of plant height and number of total tillers hill $^{-1}$ which resulted in differences of straw yield. The highest harvest index 
(44.14\%) was found in $50 \%$ of recommended dose of prilled urea and $\mathrm{P}, \mathrm{K}, \mathrm{S}, \mathrm{Zn}+$ poultry manure $5 \mathrm{t} \mathrm{ha}^{-1}$ while the lowest one (41.92\%) was found in USG $1.8 \mathrm{~g} / 4$ hills and $\mathrm{P}, \mathrm{K}, \mathrm{S}, \mathrm{Zn}+$ poultry manure $2.5 \mathrm{t} \mathrm{ha}^{-1}$.

Effect of interaction between variety and nutrient management Crop characters, yield components and yield were significantly influenced by the interaction between variety and nutrient management (Table 3). The highest number of total tillers hill ${ }^{-1}$ (12.42), number of effective tillers hill ${ }^{-1}$ (10.20), number of noneffective tillers hill ${ }^{-1}(2.17)$, grain yield $\left(6.45 \mathrm{t} \mathrm{ha}^{-1}\right)$ and straw yield $\left(9.57 \mathrm{t} \mathrm{ha}^{-1}\right)$ were obtained in BRRI dhan72 fertilized with
USG $1.8 \mathrm{~g} / 4$ hills and $\mathrm{P}, \mathrm{K}, \mathrm{S}, \mathrm{Zn}+$ poultry manure $2.5 \mathrm{t} \mathrm{ha}^{-1}$. The lowest number of total tillers hill $^{-1}$ (7.93) was found in BRRI dhan70 fertilized with poultry manure $5 \mathrm{t} \mathrm{ha}^{-1}$ while the lowest number of effective tillers hill ${ }^{-1}$ (6.67), grain yield $\left(3.5 \mathrm{t} \mathrm{ha}^{-1}\right)$ and straw yield (4.85 $\mathrm{t} \mathrm{ha}^{-1}$ ) were found in the combination of BRRI dhan70 and poultry manure $5 \mathrm{t} \mathrm{ha}^{-1}$. The highest harvest index (47.28\%) was found in the combination of BRRI dhan70 and $75 \%$ of recommended dose of prilled urea and $\mathrm{P}, \mathrm{K}, \mathrm{S}, \mathrm{Zn}+$ poultry manure $2.5 \mathrm{t} \mathrm{ha}^{-1}$ while the lowest harvest index (38.90\%) was found in the combination of BRRI dhan72 and $75 \%$ of recommended dose of prilled urea and $\mathrm{P}, \mathrm{K}, \mathrm{S}, \mathrm{Zn}+$ poultry manure $2.5 \mathrm{t} \mathrm{ha}^{-1}$.

Table 1. Effect of variety on crop characters, yield components and yield of transplant Aman rice.

\begin{tabular}{|c|c|c|c|c|c|c|c|c|c|c|c|}
\hline Variety & $\begin{array}{l}\text { Plant } \\
\text { height } \\
\text { (cm) }\end{array}$ & $\begin{array}{l}\text { No. of } \\
\text { total } \\
\text { tillers } \\
\text { hill }^{-1}\end{array}$ & $\begin{array}{l}\text { No. of } \\
\text { effective } \\
\text { tillers } \\
\text { hill }^{-1}\end{array}$ & $\begin{array}{l}\text { No. of non- } \\
\text { effective } \\
\text { tillers hill }^{-1}\end{array}$ & $\begin{array}{c}\text { Panicle } \\
\text { length } \\
\text { (cm) }\end{array}$ & $\begin{array}{l}\text { No. of } \\
\text { grains } \\
\text { panicle }^{-1}\end{array}$ & $\begin{array}{c}\text { No. of } \\
\text { sterile } \\
\text { spikelets } \\
\text { panicle }^{-1}\end{array}$ & $\begin{array}{l}1000^{-} \\
\text {grain } \\
\text { weight } \\
\text { (g) }\end{array}$ & $\begin{array}{l}\text { Grain } \\
\text { yield } \\
\left(\mathrm{t} \mathrm{ha}^{-1}\right)\end{array}$ & $\begin{array}{c}\text { Straw } \\
\text { yield } \\
\left(\mathrm{t} \mathrm{ha}^{-1}\right)\end{array}$ & $\begin{array}{c}\text { Harvest } \\
\text { index } \\
(\%)\end{array}$ \\
\hline $\begin{array}{l}\text { BRRI } \\
\text { dhan70 }\end{array}$ & $139.9 a$ & $8.74 d$ & 7.67c & $1.09 \mathrm{~b}$ & $24.74 b$ & $75.52 d$ & $20.06 a$ & $21.38 c$ & $4.84 d$ & $5.89 d$ & 45.00a \\
\hline $\begin{array}{l}\text { BRRI } \\
\text { dhan71 }\end{array}$ & $122.2 \mathrm{c}$ & $9.06 c$ & $7.76 c$ & $1.29 a$ & $23.57 b$ & $79.99 c$ & $18.22 b$ & $26.27 b$ & $5.09 c$ & $6.28 c$ & 44.83a \\
\hline $\begin{array}{l}\text { BRRI } \\
\text { dhan72 }\end{array}$ & 128.0b & $10.25 a$ & $8.85 a$ & $1.40 a$ & $24.17 b$ & 94.23a & $11.61 d$ & 27.81a & $5.88 a$ & 8.83a & $40.08 b$ \\
\hline $\begin{array}{l}\text { BRRI } \\
\text { dhan73 }\end{array}$ & $121.8 \mathrm{c}$ & $9.7 \mathrm{~b}$ & $8.31 b$ & $1.39 a$ & $26.60 a$ & $88.21 b$ & $13.59 c$ & $26.66 b$ & $5.3 b$ & $6.71 b$ & 44.35a \\
\hline Sx & 1.45 & 0.09 & 0.08 & 0.04 & 0.39 & 0.73 & 0.28 & 0.19 & 0.054 & 0.08 & 0.36 \\
\hline $\begin{array}{l}\text { Level of } \\
\text { significance }\end{array}$ & $* *$ & $* *$ & $* *$ & $* *$ & $* *$ & $* *$ & $* *$ & $* *$ & $* *$ & $* *$ & $* *$ \\
\hline CV (\%) & 4.83 & 4.25 & 4.19 & 11.85 & 6.81 & 3.68 & 7.50 & 3.10 & 4.41 & 5.12 & 3.55 \\
\hline
\end{tabular}

Table 2. Effect of nutrient management on crop characters, yield components and yield of transplant Aman rice.

\begin{tabular}{|c|c|c|c|c|c|c|c|c|c|c|c|}
\hline $\begin{array}{l}\text { Nutrient } \\
\text { management }\end{array}$ & $\begin{array}{l}\text { Plant } \\
\text { height } \\
\text { (cm) }\end{array}$ & $\begin{array}{l}\text { No. of } \\
\text { total } \\
\text { tillers } \\
\text { hill }^{-1}\end{array}$ & $\begin{array}{l}\text { No. of } \\
\text { effective } \\
\text { tillers } \\
\text { hill }^{-1}\end{array}$ & $\begin{array}{l}\text { No. of } \\
\text { non- } \\
\text { effective } \\
\text { tillers } \\
\text { hill }^{-1}\end{array}$ & $\begin{array}{c}\text { Panicle } \\
\text { length } \\
\text { (cm) }\end{array}$ & $\begin{array}{l}\text { No. of } \\
\text { grains } \\
\text { panicle }^{-1}\end{array}$ & $\begin{array}{c}\text { No. of } \\
\text { sterile } \\
\text { spikelets } \\
\text { panicle }^{-1}\end{array}$ & $\begin{array}{l}1000^{-} \\
\text {grain } \\
\text { weight } \\
\text { (g) }\end{array}$ & $\begin{array}{c}\text { Grain } \\
\text { yield } \\
\left(\mathrm{t} \mathrm{ha}^{-1}\right)\end{array}$ & $\begin{array}{c}\text { Straw } \\
\text { yield } \\
\left(\mathrm{t} \mathrm{ha}^{-1}\right)\end{array}$ & $\begin{array}{c}\text { Harvest } \\
\text { index } \\
(\%)\end{array}$ \\
\hline $\mathrm{F}_{0}$ & $122.3 b$ & $8.21 f$ & $7.15 f$ & $1.07 c$ & 24.14 & $78.12 d$ & 19.38a & $24.24 \mathrm{e}$ & $4.45 f$ & $5.76 \mathrm{e}$ & $43.76 a$ \\
\hline$F_{1}$ & 126.7ab & $9.21 d$ & $8.02 d$ & $1.19 c$ & 25.29 & $84.02 b$ & $15.64 c$ & $25.16 d$ & $5.2 d$ & $6.8 c$ & $43.43 a$ \\
\hline $\mathrm{F}_{2}$ & 127.8a & $10.12 b$ & $8.63 b$ & $1.5 \mathrm{a}$ & 24.43 & $86.03 b$ & $14.59 d$ & $26.23 b$ & $5.65 b$ & $7.26 b$ & $44.12 \mathrm{a}$ \\
\hline $\mathrm{F}_{3}$ & $129.4 a$ & $8.73 e$ & $7.58 \mathrm{e}$ & $1.14 \mathrm{c}$ & 24.55 & $80.85 c$ & $16.84 b$ & $24.95 d$ & $4.93 e$ & $6.31 d$ & $44.14 a$ \\
\hline $\mathrm{F}_{4}$ & $130.6 a$ & $9.67 c$ & $8.33 c$ & $1.33 b$ & 25.40 & $85.20 b$ & $15.31 \mathrm{~cd}$ & $25.79 b$ & $5.45 c$ & $7.04 b c$ & $44.02 \mathrm{a}$ \\
\hline $\mathrm{F}_{5}$ & 131.0a & $10.67 a$ & $9.13 a$ & $1.54 a$ & 24.81 & $92.71 a$ & $13.47 \mathrm{e}$ & $26.82 a$ & $6.0 a$ & $8.35 a$ & $41.92 b$ \\
\hline Sx & 1.78 & 0.12 & 0.09 & 0.04 & 0.481 & 0.898 & 0.342 & 0.228 & 0.07 & 0.10 & 0.446 \\
\hline $\begin{array}{l}\text { Level of } \\
\text { significance }\end{array}$ & $* *$ & $* *$ & $* *$ & $* *$ & NS & $* *$ & $* *$ & $* *$ & $* *$ & $* *$ & $* *$ \\
\hline CV (\%) & 4.83 & 4.25 & 4.19 & 11.85 & 6.81 & 3.68 & 7.50 & 3.10 & 4.41 & 5.12 & 3.55 \\
\hline
\end{tabular}

In a column, figures with same letter (s) or without letter do not differ significantly whereas figures with dissimilar letter differ significantly (as per

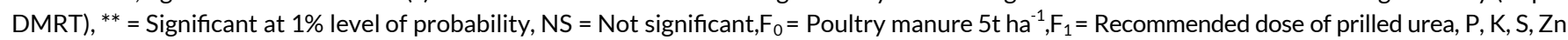
$\left(100,65,90,70,10 \mathrm{~kg} \mathrm{ha}^{-1}\right.$ of urea, TSP, MoP, Gypsum, Zinc sulphate, respectively), $\mathrm{F}_{2}=75 \%$ of recommended dose of prilled urea and $\mathrm{P}, \mathrm{K}, \mathrm{S}, \mathrm{Zn}+$ poultry manure $2.5 \mathrm{t} \mathrm{ha}^{-1}, \mathrm{~F}_{3}=50 \%$ recommended dose of prilled urea and $\mathrm{P}, \mathrm{K}, \mathrm{S}, \mathrm{Zn}+$ poultry manure $5 \mathrm{t}$ ha ${ }^{-1}, \mathrm{~F}_{4}=\mathrm{USG} 1.8 \mathrm{~g} / 4$ hills and $\mathrm{P}, \mathrm{K}, \mathrm{S}, \mathrm{Zn}$ recommended dose, $F_{5}=$ USG $1.8 \mathrm{~g}$ hill and $\mathrm{P}, \mathrm{K}, \mathrm{S}, \mathrm{Zn}+$ poultry manure $2.5 \mathrm{t} \mathrm{ha}^{-1}$. 
Table 3. Effect of interaction between variety and nutrient management on crop characters, yield components and yield of transplant Aman rice.

\begin{tabular}{|c|c|c|c|c|c|c|c|c|c|c|c|}
\hline $\begin{array}{l}\text { Interaction } \\
\text { (Variety } x \\
\text { Nutrient } \\
\text { management) }\end{array}$ & $\begin{array}{l}\text { Plant } \\
\text { height } \\
\text { (cm) }\end{array}$ & $\begin{array}{l}\text { No. of } \\
\text { total } \\
\text { tillers } \\
\text { hill }^{-1}\end{array}$ & $\begin{array}{l}\text { No. of } \\
\text { effective } \\
\text { tillers hill }\end{array}$ & $\begin{array}{l}\text { No. of } \\
\text { non- } \\
\text { effective } \\
\text { tillers } \\
\text { hill }^{-1}\end{array}$ & $\begin{array}{l}\text { Panicle } \\
\text { length } \\
\text { (cm) }\end{array}$ & $\begin{array}{c}\text { No. of } \\
\text { grains } \\
\text { panicle }^{-1}\end{array}$ & $\begin{array}{l}\text { No. of } \\
\text { sterile } \\
\text { spikelets } \\
\text { panicle }^{-1}\end{array}$ & $\begin{array}{l}1000^{-} \\
\text {grain } \\
\text { weight } \\
\text { (g) }\end{array}$ & $\begin{array}{l}\text { Grain } \\
\text { yield } \\
\left(\mathrm{t} \mathrm{ha}^{-1}\right)\end{array}$ & $\begin{array}{l}\text { Straw } \\
\text { yield } \\
\left(\mathrm{t} \mathrm{ha}^{-1}\right)\end{array}$ & $\begin{array}{l}\text { Harvest } \\
\text { index (\%) }\end{array}$ \\
\hline$\overline{V_{1} \times F_{0}}$ & 125.0 & $7.91 \mathrm{i}$ & 6.671 & $1.25 \mathrm{efg}$ & 24.75 & 66.93 & $22.92 a$ & 20.10 & $3.5 \mathrm{j}$ & 4.851 & $42.32 \mathrm{dg}$ \\
\hline $\mathrm{V}_{1} \times \mathrm{F}_{1}$ & 144.4 & $8.58 \mathrm{hi}$ & 7.7ghij & $0.83 \mathrm{ij}$ & 25.97 & 76.36 & $19.97 b c$ & 21.31 & $4.71 \mathrm{hi}$ & $5.77 \mathrm{gk}$ & $44.73 \mathrm{~cd}$ \\
\hline $\mathrm{V}_{1} \times \mathrm{F}_{2}$ & 142.2 & $9.00 \mathrm{fgh}$ & 7.92ghi & 1.08ghi & 24.42 & 77.31 & $19.03 c$ & 22.07 & $5.35 \mathrm{ef}$ & 5.93hij & $47.44 a$ \\
\hline$V_{1} \times F_{3}$ & 142.0 & $8.42 \mathrm{hi}$ & $7.25 \mathrm{jkl}$ & $1.17 \mathrm{gh}$ & 24.53 & 71.95 & $22.84 a$ & 20.70 & 4.56hi & 5.52ijk & $45.28 b d$ \\
\hline $\mathrm{V}_{1} \times \mathrm{F}_{4}$ & 143.9 & 8.83gh & $7.91 \mathrm{fgi}$ & $0.92 \mathrm{hij}$ & 24.13 & 76.50 & $19.58 \mathrm{bc}$ & 21.43 & $5.25 \mathrm{fg}$ & 5.86hij & $47.28 a$ \\
\hline $\mathrm{V}_{1} \times \mathrm{F}_{5}$ & 141.9 & 9.67def & 8.33efg & 1.33def & 24.63 & 84.07 & $16.03 e$ & 22.67 & 5.59def & $7.42 \mathrm{~cd}$ & 42.98def \\
\hline $\mathrm{V}_{2} \times \mathrm{F}_{0}$ & 122.5 & $7.93 i$ & $7.0 \mathrm{kl}$ & $0.93 \mathrm{hij}$ & 22.55 & 73.50 & $21.35 a b$ & 25.20 & $4.4 \mathrm{i}$ & $5.14 \mathrm{kl}$ & $46.18 a b$ \\
\hline $\mathrm{V}_{2} \times \mathrm{F}_{1}$ & 121.4 & 8.67hi & 7.83hij & $0.83 i j$ & 24.28 & 79.57 & $18.28 \mathrm{~cd}$ & 25.89 & $4.90 \mathrm{gh}$ & $6.23 \mathrm{fgh}$ & 44.0cde \\
\hline $\mathrm{V}_{2} \times \mathrm{F}_{2}$ & 122.6 & 9.83de & 8.0fgh & $1.75 b$ & 24.08 & 81.57 & $16.25 \mathrm{de}$ & $z 26.79$ & 5.50def & $6.7 \mathrm{ef}$ & $44.81 \mathrm{bd}$ \\
\hline $\mathrm{V}_{2} \times \mathrm{F}_{3}$ & 119.2 & $8.5 \mathrm{hi}$ & 7.33ijk & 1.17efgh & 22.75 & 75.68 & $19.52 \mathrm{bc}$ & 25.90 & $4.58 \mathrm{hi}$ & 5.72hijk & $44.47 \mathrm{de}$ \\
\hline $\mathrm{V}_{2} \times \mathrm{F}_{4}$ & 121.6 & 9.42efg & 8.0fgh & $1.42 \mathrm{cde}$ & 23.78 & 80.99 & $18.15 \mathrm{~cd}$ & 26.47 & $5.28 \mathrm{fg}$ & 6.30fgh & $45.58 \mathrm{abc}$ \\
\hline$V_{2} \times F_{5}$ & 125.7 & $10.00 \mathrm{de}$ & 8.33efg & $1.67 \mathrm{bc}$ & 24.00 & 88.65 & $15.76 \mathrm{e}$ & 27.39 & $5.88 \mathrm{bcd}$ & $7.51 \mathrm{~cd}$ & 43.92de \\
\hline $\mathrm{V}_{3} \times \mathrm{F}_{0}$ & 124.1 & $8.58 \mathrm{hi}$ & $7.66 \mathrm{hij}$ & $0.92 \mathrm{hij}$ & 23.13 & 87.52 & 14.38ef & 25.85 & 5.31efg & $7.74 c$ & 40.68gh \\
\hline $\mathrm{V}_{3} \times \mathrm{F}_{1}$ & 125.9 & $9.92 \mathrm{de}$ & 8.66cde & $1.25 \mathrm{efg}$ & 23.27 & 91.66 & 11.43gh & 27.44 & $5.93 \mathrm{~cd}$ & $8.91 b$ & 39.94gh \\
\hline $\mathrm{V}_{3} \times \mathrm{F}_{2}$ & 126.7 & $11.25 \mathrm{~b}$ & $9.41 \mathrm{~b}$ & $1.83 \mathrm{~b}$ & 23.39 & 94.61 & $10.57 \mathrm{~h}$ & 28.77 & $6.00 \mathrm{bc}$ & $9.43 a b$ & $38.90 \mathrm{~h}$ \\
\hline $\mathrm{V}_{3} \times \mathrm{F}_{3}$ & 129.0 & $9.08 \mathrm{gh}$ & $8.41 \mathrm{ef}$ & $0.67 j$ & 25.46 & 90.57 & $12.00 \mathrm{gh}$ & 27.21 & $5.66 \mathrm{def}$ & $8.03 c$ & 41.70fgh \\
\hline $\mathrm{V}_{3} \times \mathrm{F}_{4}$ & 135.4 & $10.25 \mathrm{~cd}$ & $8.66 \mathrm{cde}$ & $1.58 \mathrm{bcd}$ & 26.18 & 94.06 & $10.82 \mathrm{gh}$ & 28.47 & $5.95 \mathrm{bc}$ & 9.30ab & $39.03 \mathrm{~h}$ \\
\hline$V_{3} \times F_{5}$ & 126.8 & $12.42 \mathrm{a}$ & $10.20 \mathrm{a}$ & $2.17 a$ & 23.59 & 106.9 & $10.44 \mathrm{~h}$ & 29.12 & $6.453 a$ & $9.57 a$ & $40.2 \mathrm{fgh}$ \\
\hline $\mathrm{V}_{4} \times \mathrm{F}_{0}$ & 117.7 & $8.41 \mathrm{hi}$ & $7.25 \mathrm{jkl}$ & $1.17 \mathrm{fgh}$ & 26.13 & 84.53 & $18.8 c$ & 25.79 & $4.49 \mathrm{hi}$ & 5.30jkl & $45.86 a b c$ \\
\hline $\mathrm{V}_{4} \times \mathrm{F}_{1}$ & 114.9 & 9.66def & 7.8hij & $1.83 \mathrm{~b}$ & 27.64 & 88.50 & $12.87 \mathrm{fg}$ & 26.01 & $5.26 \mathrm{fg}$ & $6.42 \mathrm{fg}$ & $45.02 \mathrm{~cd}$ \\
\hline $\mathrm{V}_{4} \times \mathrm{F}_{2}$ & 119.5 & $10.4 \mathrm{~cd}$ & $9.08 \mathrm{bc}$ & 1.33def & 25.82 & 90.64 & $12.5 \mathrm{fgh}$ & 27.28 & 5.73 bce & $6.91 \mathrm{de}$ & $45.32 \mathrm{~cd}$ \\
\hline $\mathrm{V}_{4} \times \mathrm{F}_{3}$ & 127.7 & 8.91gh & 7.33ijk & $1.58 \mathrm{bcd}$ & 25.45 & 85.19 & $13.0 \mathrm{~g}$ & 25.99 & 4.91gh & $5.97 \mathrm{hi}$ & $45.11 \mathrm{~cd}$ \\
\hline $\mathrm{V}_{4} \times \mathrm{F}_{4}$ & 121.7 & 10.17de & $8.75 \mathrm{~cd}$ & $1.42 \mathrm{de}$ & 27.51 & 89.25 & $12.68 \mathrm{gh}$ & 26.79 & 5.32efg & 6.72ef & $44.21 \mathrm{de}$ \\
\hline$V_{4} \times F_{5}$ & 129.4 & $10.58 \mathrm{c}$ & $9.58 \mathrm{~b}$ & 1.00ghi & 27.03 & 91.15 & $11.6 \mathrm{gh}$ & 28.11 & $6.08 a b$ & $8.91 \mathrm{~b}$ & 40.56gh \\
\hline Sx & 3.57 & 0.23 & 0.19 & 0.09 & 0.97 & 1.8 & 0.69 & 0.46 & 0.13 & 0.20 & 0.89 \\
\hline Level of sig. & NS & $* *$ & $* *$ & $* *$ & NS & NS & $* *$ & NS & . & $* *$ & $* *$ \\
\hline CV (\%) & 4.83 & 4.25 & 4.19 & 11.85 & 6.81 & 3.68 & 7.50 & 3.10 & 4.41 & 5.12 & 3.55 \\
\hline
\end{tabular}

In a column, figures with same letter (s) or without letter do not differ significantly whereas figures with dissimilar letter differ significantly (as per DMRT), ${ }^{* *}=$ Significant at $1 \%$ level of probability, NS = Not significant, $\mathrm{V}_{1}=\mathrm{BRRI}$ dhan70, $\mathrm{V}_{2}=\mathrm{BRRI}$ dhan71, $\mathrm{V}_{3}=\mathrm{BRRI}$ dhan73, $\mathrm{V}_{4}=\mathrm{BRRI}$ dhan74, $F_{0}=$ Poultry manure $5 \mathrm{t} \mathrm{ha}^{-1}, \mathrm{~F}_{1}=$ Recommended dose of prilled urea, $\mathrm{P}, \mathrm{K}, \mathrm{S}, \mathrm{Zn}\left(100,65,90,70,10 \mathrm{~kg} \mathrm{ha}^{-1}\right.$ of urea, TSP, MoP, Gypsum, Zinc sulphate, respectively), $\mathrm{F}_{2}=75 \%$ of recommended dose of prilled urea and $\mathrm{P}, \mathrm{K}, \mathrm{S}, \mathrm{Zn}+$ poultry manure $2.5 \mathrm{t} \mathrm{ha}{ }^{-1}, \mathrm{~F}_{3}=50 \%$ recommended dose of prilled urea and $\mathrm{P}, \mathrm{K}, \mathrm{S}, \mathrm{Zn}+$ poultry manure $5 \mathrm{t} \mathrm{ha}^{-1}, \mathrm{~F}_{4}=$ USG $1.8 \mathrm{~g} / 4$ hills and $\mathrm{P}, \mathrm{K}, \mathrm{S}, \mathrm{Zn}$ recommended dose, $\mathrm{F}_{5}=\mathrm{USG} 1.8 \mathrm{~g}$ hill and $\mathrm{P}, \mathrm{K}, \mathrm{S}, \mathrm{Zn}+$ poultry manure $2.5 \mathrm{tha}^{-1}$.

\section{Conclusion}

Result revealed that the highest grain yield was produced in BRRI dhan72 compare to other tested varieties. In case of nutrient management, the highest grain yield was obtained when the crop was fertilized with USG $1.8 \mathrm{~g} / 4$ hills and $\mathrm{P}, \mathrm{K}, \mathrm{S}, \mathrm{Zn}+$ poultry manure $2.5 \mathrm{t} \mathrm{ha}^{-1}$. BRRI dhan72 fertilized with USG $1.8 \mathrm{~g} / 4$ hills and $\mathrm{P}, \mathrm{K}, \mathrm{S}, \mathrm{Zn}+$ poultry manure $2.5 \mathrm{t} \mathrm{ha}^{-1}$ showed the best performance in respect of grain yield. It can be concluded that transplant Aman rice BRRI dhan72 fertilized with USG $1.8 \mathrm{~g} / 4$ hills and $\mathrm{P}, \mathrm{K}, \mathrm{S}, \mathrm{Zn}+$ poultry manure $2.5 \mathrm{t} \mathrm{ha}^{-1}$ appeared as the promising practice in terms of grain yield.

Open Access: This is open access article distributed under the terms of the Creative Commons Attribution License, which permits unrestricted use, distribution, and reproduction in any medium, provided the original author(s) and the source are credited.

\section{REFERENCES}

BBS, Bangladesh Bureau of Statistics (2016). The Yearbook of Agricultural Statistics of Bangladesh Stat. Div., Minis. Plan. Govt. People's Repub., Bangladesh, Dhaka.pp.54.

Biswas, T., Paul, S.K., Sarkar, M.A.R. and Sarkar, S.K. (2016).
Integrated use of poultry manure with prilled urea and urea super granules for improving yield and protein content of aromatic rice (cv. BRRI dhan50). Progressive Agriculture, 27 (2): 86-93.

Chowdhury, S.A., Paul, S.K. and Sarkar M.A.R. (2016).Yield performance of fine aromatic rice in response to variety and level of nitrogen. Journal of Environmental Science and Natural Resources, 9 (1): 41-45.

Chen, X.P., Cui, Z.L., Vitousek, P.M., Cassman, K.G., Matson, P. A., Bai, J.S., Meng, Q.F., Hou, P., Yue, S.C., Römheld, V. and Zhang, F.S. (2011). Integrated soil crop system management for food security. Proceedings of the Natural Academy of Sciences USA, 108(16): 6399-404.

Gomez, K.A. and Gomez, A.A. (1984). Statistical Procedure for Agricultural Research. Int. Rice Res. Inst., John Wiley and Sons. New York, Chichester, Brisbance. Toronto, Singapore, pp. 139-240.

Gupta, V. and Sharma, R.S. (2007). Saving of costly fertilizers through long term application of INM in rice (Oryza sativa L.)-wheat (Triticum aestivum L.) Cropping Research on Crops, 8: 41-5.

Islam, S.M.M., Paul, S.K. and Sarkar, M.A.R. (2015). Effect of weeding regime and integrated nutrient management on yield contributing characters and yield of BRRI dhan49. 
Journal of Crop and Weed, 11: 193-197.

Jisan, M.T., Paul, S.K. and Salim, M. (2014).Yield performance of some transplant aman rice varieties as influenced by different levels of nitrogen. Journal of the Bangladesh Agricultural University, 12 (2): 321-324.

Kumar, J. and Yadav, M.P. (2008). Effect of integrated nutrient management on growth, yield attributes, yield and economics of hybrid rice (Oryza sativa L.). Research on Crops, 9: 10-3.

Nuruzzaman, M., Yamamoto, Y., Nitta, Y., Yoshida, T. and Miyazaki, A. (2000). Varietal differences in tillering ability of fourteen Japonica and Indica rice varieties. Soil Science and Plant Nutrition, 46 (2): 381-391.

Pal, S., Paul, S.K., Sarkar, M.A.R and Gupta, D.R. (2016). Response on yield and protein content of aromatic fine rice varieties to integrated use of cowdung and inorganic fertilizers. Journal of Crop and Weed, 12(1): 01-06.

Roy, B., Sarkar, M.A.R and Paul, S.K. (2015). Effect of integrated nutrient management in Boro rice cultivation. SAARC Journal of Agriculture, 13 (2): 131-140.
Sarkar, S.K., Sarkar, M.A.R., Islam, N. and Paul, S.K. (2014). Yield and quality of aromatic fine rice as affected by variety and nutrient management. Journal of the Bangladesh Agricultural University, 12 (2): 279-284.

Singh, Y. and Singh, U.S. (2008). Genetic diversity analysis in aromatic rice germplasm using agro- morphological traits. Indian Journal of Plant Genetic Resources, 21(1): 32-37.

Sun, B., Zhang, L., Yang, L., Zhang, F., Norse, D. and Zhu, Z. (2012). Agricultural Non-Point Source Pollution in China: Causes and Mitigation Measures. Ambio, 41(4): 370-379. http:// doi.org/10.1007/s13280-012-0249-6

Tyeb, A., Paul, S. K. and Samad, M.A. (2013). Performance of variety and spacing on the yield and yield contributing characters of transplant Aman rice. Journal of Agroforestry and Environment, 7(1): 57-60.

Wu, W. and Ma, B. (2015). Integrated nutrient management (INM) for sustaining crop productivity and reducing environmental impact: A review. Science of the Total Environment, 512-513: 415-42, DOI. 10.1016/j.scitotenv.2014.12.101. 